\title{
Clostridium difficile as an Emerging Foodborne Pathogen of Public Health Significance
}

\author{
Mahendra Pal ${ }^{*}$ and Mati Roba Bulcha ${ }^{2}$ \\ ${ }^{1}$ Narayan Consultancy on Veterinary Public Health and Microbiology, Gujarat, India \\ ${ }^{2}$ Yemalog Walal Woreda Livestock and Fishery Development and Resource Office, \\ Oromia, Ethiopia \\ *Corresponding Author: Mahendra Pal, Professor, Founder Director of Narayan \\ Consultancy on Veterinary Public Health and Microbiology, Gujarat, India.
}

\author{
Received: March 29, 2021 \\ Published: April 27, 2021 \\ (C) All rights are reserved by Mahendra Pal \\ and Mati Roba Bulcha.
}

\begin{abstract}
The recent decades have witnessed the emergence and re-emergence of foodborne pathogens that cause significant morbidity and mortality worldwide. Clostridium difficile is an anaerobic, spore-forming pathogen that is a common cause of hospital and antimicrobial-associated diarrhea. Clostridium difficile has been isolated from food animals, vegetables, and retail meat that it might be a zoonotic and foodborne pathogen. Zoonotic transmission of Clostridium difficile has occurred after direct or indirect contact with contaminated animal feces. Understanding how the bacterium enters foods and humans are important for developing sciencebased prevention strategies. The presence of asymptomatic animals that bring and shed bacterium into the environment, infecting individuals or communities directly or through the food chain, is a major public health concern. The most common symptom of Clostridium difficile infections is diarrhea, which is not visibly bleeding and may be soft or watery. In addition, hypoalbuminemia, and sometimes fulminating colitis are some of the other symptoms exhibited by the patient. Since nucleic acid tests have a lower specificity, some clinicians agree that toxin enzyme immunoassays are better predictors of disease. The use of toxin gene cycle thresholds to predict the existence and absence of toxins has recently piqued interest. A variety of novel Clostridium difficile infections therapies are in different growth stages, including an antibiotic ridinilazole and RBX2660, and a vaccine is all in phase 3 trials. Certain measures like hand hygiene, isolation precautions, environmental sanitation, and limited use of broad-spectrum antibiotics may help in the prevention and control of infection due to Clostridium difficile.

Keywords: Bacterium; Clostridium difficile; Diarrhoea; Emerging Foodborne Pathogen; Hand Hygiene; Public Health; Zoonotic Transmission
\end{abstract}

\section{Introduction}

For public health agencies and organizations, the emergence and re-emergence of foodborne pathogens is a constant concern [1]. Changes in the behavior of microorganisms and consumers, changes in agricultural practices, and climate changes are contributing to the emergence of foodborne pathogens. After acquiring new virulence factors, foodborne pathogens can re-emerge as more dangerous. Previously established foodborne pathogens will re-emerge as more virulent pathogens after gaining new virulence factors, such as antibiotic resistance determinants [2].

Clostridium difficile is an anaerobic, spore forming pathogen that is a common cause of hospital and antimicrobial associated diarrhea. In humans and animals, Clostridium difficile is an important gastrointestinal pathogen [3]. Recent studies have demonstrated the isolation of Clostridium difficile from foods mostly in the developed country and meat products intended for consumption by pets [4].

Clostridium difficile infection in humans has not been proven to be transmitted directly from animals, food, or the environment. Several studies have found Clostridium difficile in a variety of foods, including meat, raw milk, vegetables, and seafood, supporting the theory that spore-contaminated foods could be contributing to Clostridium difficile exposure and transmission [5]. The presence of Clostridium difficile in animals and common PCR ribotypes between humans and animals help zoonotic transmission [6]. 
The presence of asymptomatic animals that bring and shed the bacterium into the environment, infecting individuals or communities directly or through the food chain, is a major public health concern [7]. Clostridium difficulties the pathogen that causes the most healthcare-associated infections in the US, with an estimated 500,000 infections and 29,000 deaths each year [8]. The most common symptom of CDI is diarrhea, which is not visibly bleeding and may be soft or watery. Fever, leukocytosis, stomach pain, ileus, hypoalbuminemia and sometimes fulminant colitis are some of the other symptoms exhibited by the patient [9].

Clostridium difficile testing is usually done using an enzyme immunoassay procedure in most laboratories [10]. A variety of novel CDI therapies are in different growth stages. The antibiotic ridinilazole, the microbiome products SER-109 and RBX2660, and a vaccine are all in phase 3 trials currently. Probiotics, especially Saccharomyces boulardii and more recently, fecal microbiota transplantation, have emerged as effective forms of prevention and therapy. Understanding how Clostridium difficile enters foods and humans are important for developing science-based prevention strategies [11]. The present communication delineates the emerging role of Clostridium difficile as an emerging foodborne pathogen of public health concern.

\section{Clostridium difficile in food-producing animals}

Clostridium difficile produces resistant spores that allow longterm survival and dispersal in the health-care system and the wider environment [12]. According to molecular characterization and epidemiological research, the zoonotic risk for Clostridium difficile associated with dogs and cats in the population is minimal. Recent reports of Clostridium difficile colonization and infection in dogs suggest that Clostridium difficile may be a pathogen that affects small pets [13].

Clostridium difficile has been widely described both in diarrheic and healthy pigs. In the early 1980s, Clostridium difficile was discovered in cattle as an intestinal contaminant. Clostridium difficile in ruminants had not been well studied until recently. Furthermore, the most common manifestation of cattle CDI is pre-weaning neonatal calf enteritis with a high mortality rate [7]. In poultry, necrotizing enteritis, acute onset diarrhea and rapid progression to death are the clinical manifestations of CDI infection. Infected birds typically die within three days of showing symptoms. The mortality rates of CDI-infected poultry are usually very high [14].
The bacterium's invasion of healthy sheep and goats promotes animal-to-animal transmission as well as zoonotic transmission. Sheep and goats have primarily been identified as asymptomatic carriers of Clostridium difficile and infection appears to decrease with age in sheep and goats [7].

\section{Diagnosis and treatment of Clostridium difficile infections}

Clostridium difficile-associated disease (CDAD) is diagnosed in the laboratory using fecal specimens for culture and toxin detection. Culture is carried out on a selective media that is commercially available. When viewed under a dissecting microscope, the morphology of Clostridium difficile colonies is typical. Gas-liquid chromatography is the best method for obtaining definitive identification [2]. Nucleic acid tests are the method of choice in approximately $50 \%$ of laboratories in the United States, and they are the most sensitive and least complex methods for detecting Clostridium difficile in the stool. Since nucleic acid tests have a lower specificity, some clinicians agree that toxin enzyme immunoassays [15], despite their poor performance in some patient populations, are better predictors of disease [10].

The cell cytotoxicity neutralization assay (CCNA), toxigenic culture (TC), toxin AB enzyme immunoassay (toxin AB EIA), glutamate dehydrogenase (GDH) assay and nucleic acid amplification tests are currently available for diagnosing Clostridium difficile infections [16]. Over diagnosis of Clostridium difficile infection (CDI) occurs when nucleic acid amplification tests (NAATs) are used to diagnose the infection. To enhance the clinical specificity of NAATs, the use of toxin gene cycle thresholds (CTs) to predict the existence and absence of toxins have recently piqued interest [17].

\section{Prevention and Control}

It is extremely difficult to eradicate Clostridium difficile spores from the farrowing environment because they are resistant to most disinfectants, heat, and ultraviolet light. As a result, veterinarians who are dealing with neonatal infections should keep this in mind [18]. It is important to use limited antibiotics, strict enteric measures while caring for diarrhea patients, and thorough cleaning of clinical areas for the prevention of the disease. Generally, hand hygiene, isolation precautions, environmental sanitation, and limited use of broad-spectrum antibiotics are all strategies for preventing and controlling Clostridium difficile infection [12]. 


\section{Conclusions and Recommendations}

Clostridium difficile has remained a major source of concern in hospitals and a major research subject around the world. Animals and humans may be colonized with similar Clostridium difficile clones or strains that cluster in the same lineage. Clostridium difficile be classified as a zoonotic pathogen, with the possibility of interspecies transmission and the presence of a common contamination source with animals serving as a reservoir for humans. The epidemiology of Clostridium difficile infection is changing, with a rise in disease incidence and severity, the emergence of a new outbreak strain, and an apparent increase in infections among community residents. There are several unanswered questions regarding Clostridium difficile epidemiology, so it is important to keep an eye on ongoing studies to see whether it poses a danger as a foodborne pathogen. Based on the above conclusion the following recommendations are forwarded:

- Good hand hygiene before entering a patient's room and after caring for a patient with CDI by washing hands with soap and water.

- One Health approaches that focus on collaborative efforts between human and animal health professionals will contribute significantly to zoonotic infectious disease management and reducing the environmental risks of Clostridium difficile.

- Public education on the zoonotic disease can be useful to decrease the effects and risks.

- $\quad$ Further studies should be conducted on the role of risk factors in the emergence and re-emergence of this emerging foodborne zoonotic pathogen.

\section{Acknowledgements}

The authors are very thankful to Prof. Dr. R.K. Narayan for his suggestions during the preparation of manuscript and Anubha Priyabandhu for computer help.

\section{Contribution of Authors}

All the authors contributed equally. They read the final version and approved it for publication.

\section{Conflict of Interest}

The authors declare that they do not have conflict of interest.

\section{Source of Financial Grant}

There was no financial support for this manuscript.

\section{Bibliography}

1. Schirone M., et al. "Foodborne pathogens: Hygiene and safety". Frontiers in Microbiology 10 (2019): 1974.

2. El-Sayed A and Kamel M. "Climatic changes and their role in emergence and re-emergence of diseases". Environmental Science and Pollution Research 27.18 (2020): 22336-22352.

3. Weese JS. "Clostridium (Clostridioides) difficile in animals". Journal of Veterinary Diagnostic Investigation 32.2 (2020): 213-221.

4. Rodriguez C., et al. "Clostridium difficile beyond stools: dog nasal discharge as a possible new vector of bacterial transmission". Heliyon 5.5 (2019): e01629.

5. Barbosa J., et al. "Methods currently applied to study the prevalence of Clostridioides difficile in foods". AIMS Agriculture and Food 5.1 (2020): 102-128.

6. Tkalec V., et al. "Clostridioides difficile in national food surveillance, Slovenia, 2015 to 2017". Eurosurveillance 25.16 (2020): 1900479.

7. Kachrimanidou M., et al. "Clostridioides (Clostridium) difficile in food-producing animals, horses, and household pets: a comprehensive review". Microorganisms 7.12 (2019): 667.

8. Turner N A., et al. "Novel and emerging sources of Clostridioides difficile infection”. Plos Pathogens 15.12 (2019): e1008125.

9. Aletaha N., et al. "Clinical and pathological features of ulcerative colitis in patients with and without Clostridium difficile infection; an observational study". Middle East Journal of Digestive Diseases 11.1 (2019): 17.

10. Carroll KC. "Tests for the diagnosis of Clostridium difficile infection: The next generation". Anaerobe 17.4 (2011): 170-174.

11. Durham S H., et al. "Navigating changes in Clostridioides difficile prevention and treatment". Journal of Managed Care and Specialty Pharmacy 26.12 (2020): S3-S23.

12. Knetsch C W., et al. "Zoonotic transfer of Clostridium difficile harboring antimicrobial resistance between farm animals and humans". Journal of Clinical Microbiology 56.3 (2018). 
13. Rabold D., et al. "The zoonotic potential of Clostridium difficile from small companion animals and their owners". Plos One 13.2 (2018): e0193411.

14. Frazier K S., et al. "Diagnosis of enteritis and enterotoxemia due to Clostridium difficile in captive ostriches (Struthio camelus)". Journal of Veterinary Diagnostic Investigation 5.4 (1993): 623-625.

15. Carroll K C and Mizusawa M. "Clostridioides difficile infection: Laboratory tests for the diagnosis of Clostridium difficile". Clinics in Colon and Rectal Surgery 33.2 (2020): 73.

16. Chung H S., et al. "Laboratory diagnosis of Clostridium difficile infection in Korea: the first national survey". Annals of Laboratory Medicine 39.3 (2019): 317.

17. Sandlund J and Wilcox M H. "Ultrasensitive detection of Clostridium difficile toxins reveals suboptimal accuracy of toxin gene cycle thresholds for toxin predictions". Journal of Clinical Microbiology 57.6 (2019).

18. Songer J G and Uzal F A. "Clostridial enteric infections in pigs". Journal of Veterinary Diagnostic Investigation 17.6 (2005): 528-536.

\section{Assets from publication with us}

- Prompt Acknowledgement after receiving the article

- Thorough Double blinded peer review

- Rapid Publication

- Issue of Publication Certificate

- High visibility of your Published work

Website: https://www.actascientific.com/

Submit Article: https://www.actascientific.com/submission.php Email us: editor@actascientific.com

Contact us: +919182824667 